\title{
Amorphization Effect for Kondo Semiconductor $\mathrm{CeRu}_{2} \mathbf{A l}_{10}$
}

\author{
Yusuke Amakai, ${ }^{1,2}$ Yasuhiro Shiojiri, ${ }^{1}$ Kei Ishihara, ${ }^{1}$ Hiroto Hitotsukabuto, ${ }^{1}$ \\ Shigeyuki Murayama, ${ }^{1}$ Naoki Momono, ${ }^{1,2}$ and Hideaki Takano' \\ ${ }^{1}$ Graduate School of Engineering, Muroran Institute of Technology, 27-1 Mizumoto-cho, Muroran, Hokkaido 050-8585, Japan \\ ${ }^{2}$ Research Center for Environmentally Friendly Materials Engineering, Muroran Institute of Technology, \\ 27-1 Mizumoto-cho, Muroran, Hokkaido 050-8585, Japan
}

Correspondence should be addressed to Yusuke Amakai; a-rain@mmm.muroran-it.ac.jp

Received 27 March 2017; Accepted 4 May 2017; Published 24 May 2017

Academic Editor: Joseph S. Poon

Copyright (C) 2017 Yusuke Amakai et al. This is an open access article distributed under the Creative Commons Attribution License, which permits unrestricted use, distribution, and reproduction in any medium, provided the original work is properly cited.

\begin{abstract}
We measured the magnetic susceptibility $\chi$, electrical resistivity $\rho$, and specific heat $C_{p}$ of a sputtered amorphous $(a-) \mathrm{CeRu}_{2} \mathrm{Al}_{10}$ alloy. $\chi$ value for $a-\mathrm{CeRu}_{2} \mathrm{Al}_{10}$ alloy follows a Curie-Weiss paramagnetic behavior in the high-temperature region, and magnetic transition was not observed down to $2 \mathrm{~K}$. The effective paramagnetic moment $p_{\text {eff }}$ is $1.19 \mu_{B} /$ Ce-atom. The resistivity shows a typical disordered alloy behavior, that is, small temperature dependence for the whole temperature range. We observed an enhancement of $\rho$ and $C_{p} / T$ in the low-temperature region of $T<10 \mathrm{~K}$. The enhancement in $\rho$ is suppressed by applying a magnetic field. It is suggested that this behavior is caused by the Kondo effect.
\end{abstract}

\section{Introduction}

The ternary rare-earth compound, $\mathrm{CeRu}_{2} \mathrm{Al}_{10}$, exhibits an unusual antiferromagnetic phase transition at $T_{0} \approx 27 \mathrm{~K}$ $[1,2]$. The resistivity for $\mathrm{CeRu}_{2} \mathrm{Al}_{10}$ exhibits a semiconducting behavior in the paramagnetic phase. However, this behavior is suppressed by substituting $\mathrm{La}$ in Ce-site of $\mathrm{CeRu}_{2} \mathrm{Al}_{10}$. In addition, $T_{0}$ decreases rapidly with an increase in La concentration and disappears when about half of $\mathrm{Ce}$ is substituted by La. The resistivity for Ce dilute region of Lasubstituted $\mathrm{CeRu}_{2} \mathrm{Al}_{10}$ exhibits a metallic behavior at high temperatures, and the resistivity exhibits a minimum in the low-temperature region. Tanida et al. proposed that the longrange magnetic order was suppressed randomly in Ce dilute region for La substitution of $\mathrm{CeRu}_{2} \mathrm{Al}_{10}$. The authors pointed out that the resistivity minimum can be explained by a typical impurity Kondo effect, where Ce exists as a magnetic impurity $[3,4]$. Moreover, the semiconducting band gap of $\mathrm{CeRu}_{2} \mathrm{Al}_{10}$ is broken by La substitution.

However, several studies on the Kondo effect of structural-disordered Ce-alloys such as bulk metallic glasses and amorphous alloys, where Ce-atom is arranged randomly, have been recently performed. For example, in Ce-Al bulk metallic glasses, the tunable competition between the Kondo effect and the Ruderman-Kittel-Kasuya-Yoshida (RKKY) interaction with the variation in Ce-concentration and the magnetic field [5] is suggested. We studied the low-temperature properties of several binary amorphous Ce-alloys. Amorphous $(a-) \mathrm{Ce}-\mathrm{Mn}$ and $a-\mathrm{Ce}-\mathrm{Ru}$ alloys in Ce highconcentration region exhibit a large electronic specific heat coefficient $\gamma\left(>200 \mathrm{~mJ} / \mathrm{molK}^{2}\right)$ and $T^{2}$ law with a large coefficient $A\left(>0.02 \mu \Omega \mathrm{cm} / \mathrm{K}^{2}\right)$ in the low-temperature resistivity [6-10]. From these results, we show that an itinerant heavyfermion state occurs at low temperatures as a Fermi-liquid ground state in the structure-disordered system after the formation of a dense Kondo state.

In this study, to investigate the influence of the structuraldisordered effect on the electrical resistivity and magnetic properties for $\mathrm{CeRu}_{2} \mathrm{Al}_{10}$, we prepared $a-\mathrm{CeRu}_{2} \mathrm{Al}_{10}$ alloy and measured its magnetic susceptibility, resistivity, and specific heat. In addition, we prepared $a-\mathrm{LaRu}_{2} \mathrm{Al}_{10}$ alloy that does not have $4 f$-electrons with a rare-earth element for comparison.

\section{Experimental}

Bulk ingots of $\mathrm{CeRu}_{2} \mathrm{Al}_{10}$ and $\mathrm{LaRu}_{2} \mathrm{Al}_{10}$ were prepared by using the arc-melting method with a stoichiometric 
composition of Ce 99.9\%, La 99.9\% (Nippon Yttrium Co., Ltd.), Ru 99.95\% (Rare Metallic Co., Ltd.), and Al 99.99\% (Mitsuwa Chemicals Co., Ltd.), in Ar atmosphere. The amorphous alloy was prepared using a dc high-rate sputtering method with arc-melt ingots on a water-cooled $\mathrm{Cu}$ substrate $(30 \mathrm{~mm} \phi)$. The sample thickness was $\sim 200 \mu \mathrm{m}$. The structure of the obtained samples was confirmed using X-ray diffraction measurements. Measurements were performed with an as-sputtered film. The chemical compositions of the present amorphous alloys were determined using scanning electron microscope energy dispersive $\mathrm{X}$-ray spectroscopy (SEM-EDS) to be $\mathrm{Ce}_{10} \mathrm{Ru}_{15} \mathrm{Al}_{75}$ and $\mathrm{La}_{8} \mathrm{Ru}_{17} \mathrm{Al}_{75}$ (suffixes represent at\%). Thus, we will use the notations $a-\mathrm{CeRu}_{2} \mathrm{Al}_{10}$ and $a$ - $\mathrm{LaRu}_{2} \mathrm{Al}_{10}$ for the samples, hereafter. The magnetic susceptibility was measured using a commercial SQUID magnetometer (Quantum Design MPMS) from 2 to $300 \mathrm{~K}$. The electrical resistivity was measured by using a typical four-terminal method (Quantum Design PPMS) from 2 to $300 \mathrm{~K}$. The resistivity measurements of $a-\mathrm{CeRu}_{2} \mathrm{Al}_{10}$ alloy were performed in a magnetic field $(0,20 \mathrm{kOe}, 40 \mathrm{kOe}$, and $60 \mathrm{kOe}$ ) and the temperature range was $2-60 \mathrm{~K}$. The specific heat was measured by PPMS from 2 to $300 \mathrm{~K}$.

\section{Results and Discussion}

Figure 1 shows the $\mathrm{X}$-ray diffraction patterns for $a$-CeRu $\mathrm{Al}_{10}$ and $a$-LaRu $\mathrm{Ll}_{10}$ alloys. The diffraction patterns for both alloys exhibit two broad peaks at the center at about $25^{\circ}$ and $42^{\circ}$, and definite Bragg peaks are not observed. Therefore, the samples are identified as amorphous materials.

Figure 2 shows the temperature dependence of the magnetic susceptibility $\chi$ (left axis) and the inverse susceptibility $1 /\left(\chi-\chi_{0}\right)$ (right axis) for $a-\mathrm{CeRu}_{2} \mathrm{Al}_{10}$ and $a-\mathrm{LaRu}_{2} \mathrm{Al}_{10}$ alloys at $H=10 \mathrm{kOe}$. We calculated $\chi$ using the composition ratio of $\mathrm{Ce}_{10} \mathrm{Ru}_{15} \mathrm{Al}_{75}$ obtained by SEM-EDS as 1 mol. $\chi$ value for $a$-CeRu $\mathrm{Al}_{10}$ alloy increases monotonically with decreasing temperature, and a magnetic transition is not observed in the measurement temperature region. However, $\chi$ value for $a$ - $\mathrm{LaRu}_{2} \mathrm{Al}_{10}$ alloy is almost independent of the temperature and is very small value $\left(<10^{-6} \mathrm{emu} / \mathrm{mol}\right) .1 /\left(\chi-\chi_{0}\right)$ value for $a-\mathrm{CeRu}_{2} \mathrm{Al}_{10}$ alloy exhibits a linear behavior in the hightemperature region of $T>30 \mathrm{~K}$ following the Curie-Weiss law,

$$
\frac{1}{\left(\chi-\chi_{0}\right)}=\frac{1}{C}(T-\theta),
$$

where $\chi_{0}$ is a constant for the independence of temperature, $C$ is the Curie constant, and $\theta$ is the Weiss temperature. $\chi_{0}$ value for $a-\mathrm{CeRu}_{2} \mathrm{Al}_{10}$ alloy obtained from (1) was $\sim 1.0 \times$ $10^{-6} \mathrm{emu} / \mathrm{mol}$. The value of $\theta$ is $-20 \mathrm{~K}$. $C$ is obtained as

$$
C=\frac{N_{\mathrm{Ce}} \mu_{\mathrm{eff}}^{2}}{3 k_{B}},
$$

where $N_{\mathrm{Ce}}$ is the number of Ce atoms, $\mu_{\text {eff }}$ is the paramagnetic effective magnetic moment, and $k_{B}$ is the Boltzmann constant. Here, $\mu_{\text {eff }}$ is calculated from Ce-concentration (10 at $\%$ ) of composition ratio $\mathrm{Ce}_{10} \mathrm{Ru}_{15} \mathrm{Al}_{75}$ obtained from SEM-EDS.
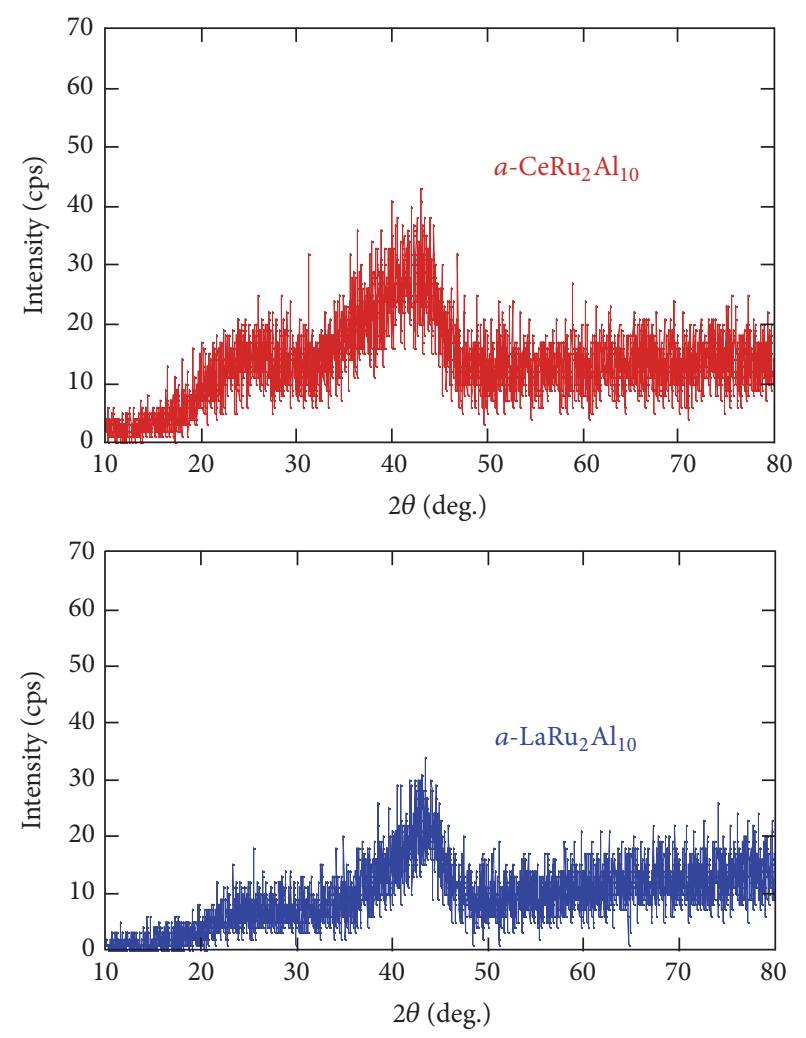

FIGURE 1: X-ray diffraction pattern of $a-\mathrm{CeRu}_{2} \mathrm{Al}_{10}$ and $a-\mathrm{LaRu}_{2} \mathrm{Al}_{10}$ alloys.

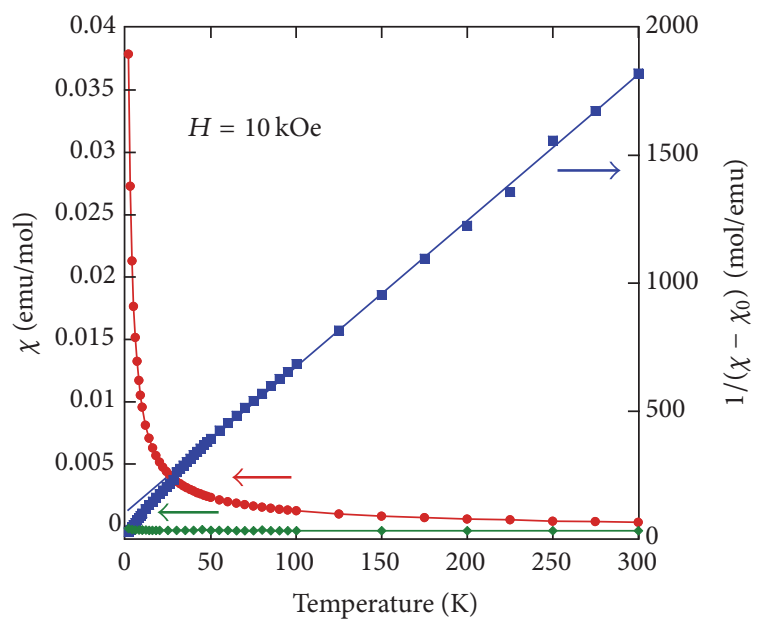

FIGURE 2: Temperature dependence of the magnetic susceptibility $\chi$ and inverse susceptibility $1 /\left(\chi-\chi_{0}\right)$ for $a-\mathrm{CeRu}_{2} \mathrm{Al}_{10}$ alloy. The green points and line are the temperature dependence of the magnetic susceptibility $\chi$ for $a-\mathrm{LaRu}_{2} \mathrm{Al}_{10}$ alloy.

The estimated $\mu_{\mathrm{eff}}$ obtained using (2) is $1.19 \mu_{B} / \mathrm{Ce}$-atom. Since the effective magnetic moment expected for trivalent $\mathrm{Ce}(J=5 / 2)$ is $2.54 \mu_{B}$, the obtained value of $1.19 \mu_{B} / \mathrm{Ce}$ is about half this value. Therefore, it is expected that about half of Ce of a-CeRu $\mathrm{Al}_{10}$ alloy exists as nonmagnetic tetravalent $\mathrm{Ce}$ and the remaining half exists as magnetic trivalent $\mathrm{Ce}$ in the alloy. 


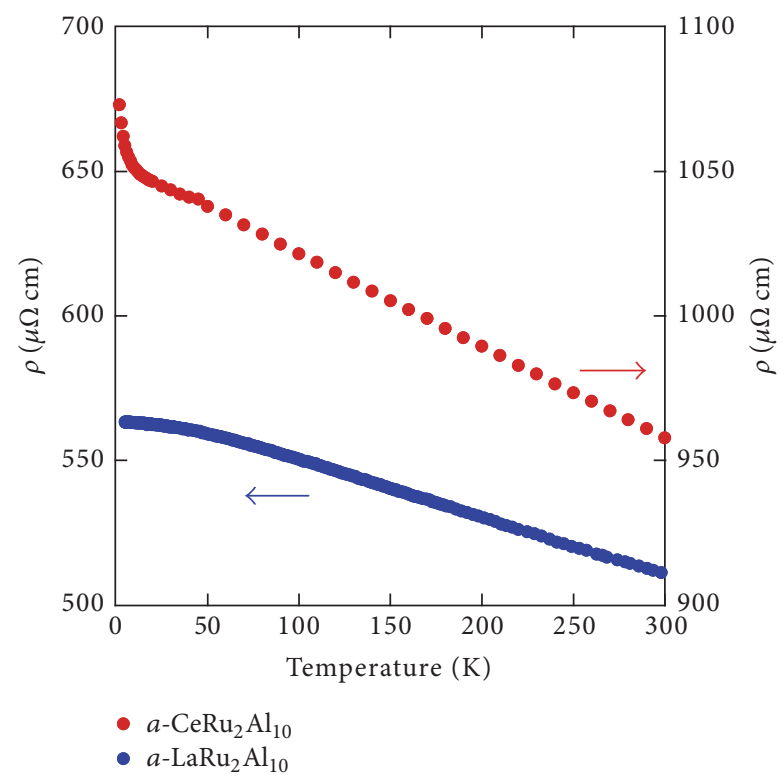

FIGURE 3: Temperature dependence of the resistivity $\rho$ for $a-\mathrm{CeRu}_{2} \mathrm{Al}_{10}$ and $a-\mathrm{LaRu}_{2} \mathrm{Al}_{10}$ alloys.

Figure 3 shows the temperature dependence of the resistivity $\rho$ for $a-\mathrm{CeRu}_{2} \mathrm{Al}_{10}$ and $a-\mathrm{LaRu}_{2} \mathrm{Al}_{10}$ alloys. The value of $\rho$ at $300 \mathrm{~K}$ for $a-\mathrm{CeRu}_{2} \mathrm{Al}_{10}$ alloy is about 2 times greater than that of $a-\mathrm{LaRu}_{2} \mathrm{Al}_{10}$ alloy. $\rho$ value for both alloys exhibits small temperature dependence less than $10 \%$ in the whole temperature region, and it increases with decreasing temperature. Such temperature dependence is one of the characteristics of disordered alloys. In contrast to $\rho$ value of $a-\mathrm{LaRu}_{2} \mathrm{Al}_{10}$ alloy, which increases monotonically with decreasing temperature, $\rho$ value of $a-\mathrm{CeRu}_{2} \mathrm{Al}_{10}$ alloy exhibits an increase in the low-temperature region. Generally, in the case of disordered alloys, the resistivity is larger than that for the crystalline counterparts owing to the random arrangement of atoms. However, the absolute value of the low-temperature $\rho$ in $a$-CeRu $\mathrm{Al}_{10}$ alloy is on about the same order as that for the polycrystalline $\mathrm{CeRu}_{2} \mathrm{Al}_{10}$. In addition, although $\rho$ value of $a-\mathrm{CeRu}_{2} \mathrm{Al}_{10}$ alloy increases with decreasing temperature, a small temperature dependence similar to that for the typical disordered alloy was observed. Therefore, it is considered that the semiconductor band for crystalline $\mathrm{CeRu}_{2} \mathrm{Al}_{10}$ exhibited a metallic behavior as a result of amorphization.

Figure 4 shows the low-temperature specific heat $C_{p}$ over $T$ versus $T^{2}$ plots for $a-\mathrm{CeRu}_{2} \mathrm{Al}_{10}$ and $a-\mathrm{LaRu}_{2} \mathrm{Al}_{10}$ alloys. The inset shows the temperature dependence of $C_{p}$ for the alloys. $C_{p}$ values of both alloys are almost in agreement in the whole temperature region, as shown in the inset. The lowtemperature specific heat of a usual metal can be expressed by

$$
C_{p}=\gamma T+\beta T^{3},
$$

where $\gamma T$ is the electronic specific heat term and $\beta T^{3}$ is the phonon specific heat term. This relation can also be applied to amorphous alloys [11]. As shown in Figure $4, C_{p} / T$ of $a-\mathrm{LaRu}_{2} \mathrm{Al}_{10}$ alloy follows a linear relation as a function

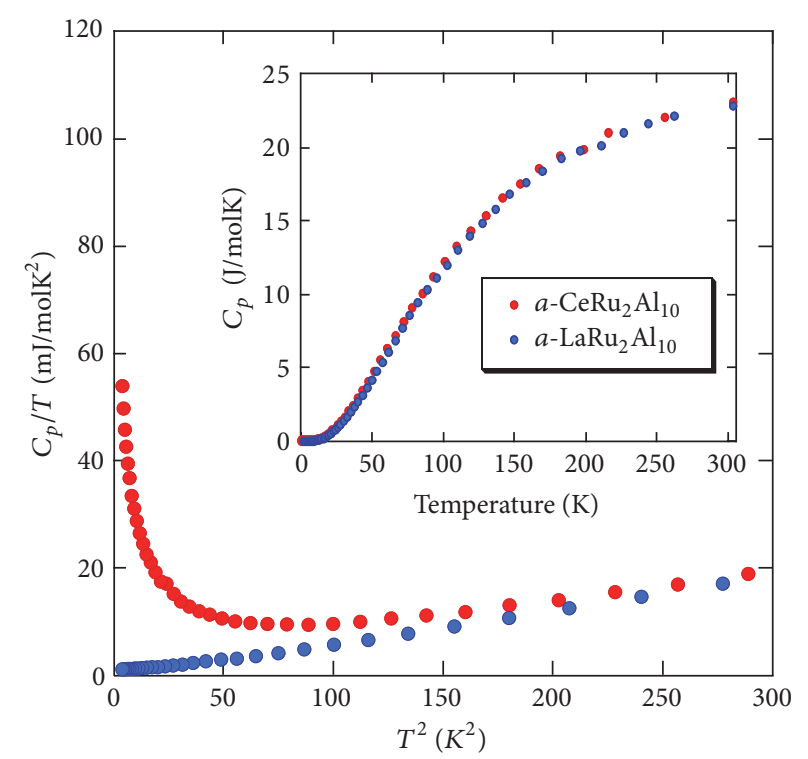

Figure 4: $C_{p} / T$ versus $T^{2}$ plot for $a-\mathrm{CeRu}_{2} \mathrm{Al}_{10}$ and $a-\mathrm{LaRu}_{2} \mathrm{Al}_{10}$ alloys. The inset shows the temperature dependence of the specific heat $C_{p}$ for $a-\mathrm{CeRu}_{2} \mathrm{Al}_{10}$ and $a-\mathrm{LaRu}_{2} \mathrm{Al}_{10}$ alloys.

of $T^{2}$ below $50 \mathrm{~K}^{2}$. However, $C_{p} / T$ of $a-\mathrm{CeRu}_{2} \mathrm{Al}_{10}$ alloy increases rapidly with decreasing $T^{2}$ below $100 \mathrm{~K}^{2}$. The value of $C_{p} / T$ at the lowest temperature is $\sim 54 \mathrm{~mJ} / \mathrm{molK}^{2}$. The temperature region where the enhancement in $C_{p} / T$ occurs agrees with the temperature region of the upturn for $\rho$. Such enhancement in $C_{p} / T$ and $\rho$ at low-temperature does not occur for $a-\mathrm{LaRu}_{2} \mathrm{Al}_{10}$. Therefore, it is considered to be due to the magnetic contribution of the $4 f$-electron of Ce. In order to clarify the magnetic contribution of the $4 f$-electron of $\mathrm{Ce}$ for $a-\mathrm{CeRu}_{2} \mathrm{Al}_{10}$, we measured the magnetoresistance of $a$ $\mathrm{CeRu}_{2} \mathrm{Al}_{10}$.

Figure 5 shows the magnetic field dependence of the transverse magnetoresistance $\Delta \rho / \rho$ at $2 \mathrm{~K}, 10 \mathrm{~K}$, and $60 \mathrm{~K}$ for $a-\mathrm{CeRu}_{2} \mathrm{Al}_{10}$ alloy. We calculated $\Delta \rho / \rho$ as follows:

$$
\frac{\Delta \rho}{\rho}=\frac{\rho(H)-\rho(0)}{\rho(0)},
$$

where $\rho(H)$ is the resistivity in the magnetic field and $\rho(0)$ is the resistivity at zero field. $\Delta \rho / \rho$ increases negative with increasing magnetic field strength. $\Delta \rho / \rho$ at $2 \mathrm{~K}$ exhibits a large negative enhancement compared with that at other temperatures. Figure 6 shows the temperature dependence of $\Delta \rho / \rho$ at $20 \mathrm{kOe}, 40 \mathrm{kOe}$, and $60 \mathrm{kOe}$ for $a-\mathrm{CeRu}_{2} \mathrm{Al}_{10}$ alloy. The $\Delta \rho / \rho$ in the high-temperature region $(T>10 \mathrm{~K})$ is almost independent of the temperature for all the magnetic fields. However, $\Delta \rho / \rho$ in the low-temperature region increases rapidly with decreasing temperature at $T<10 \mathrm{~K}$ for all the fields. Thus, the enhancement of $\rho$ at low temperatures is suppressed by the applied magnetic field.

The present $a-\mathrm{CeRu}_{2} \mathrm{Al}_{10}$ alloy seems reasonable that the magnetic order of the crystalline counterpart have disappeared because they lose a long-range crystallographic order by amorphization. However, the local structure of 


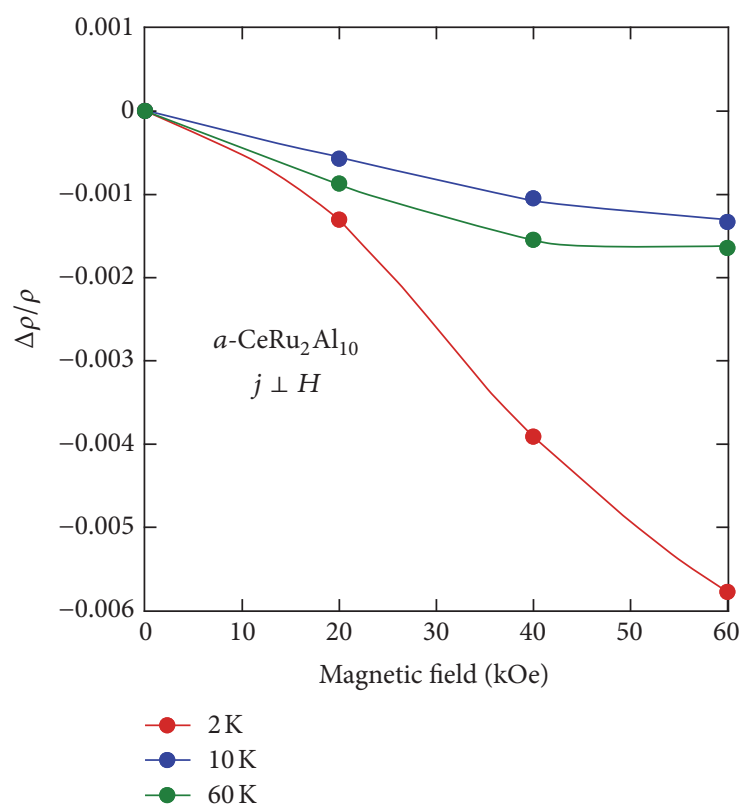

FIGURE 5: Field dependence of the magnetoresistance $\Delta \rho / \rho$ at various temperatures for $a-\mathrm{CeRu}_{2} \mathrm{Al}_{10}$ alloy.

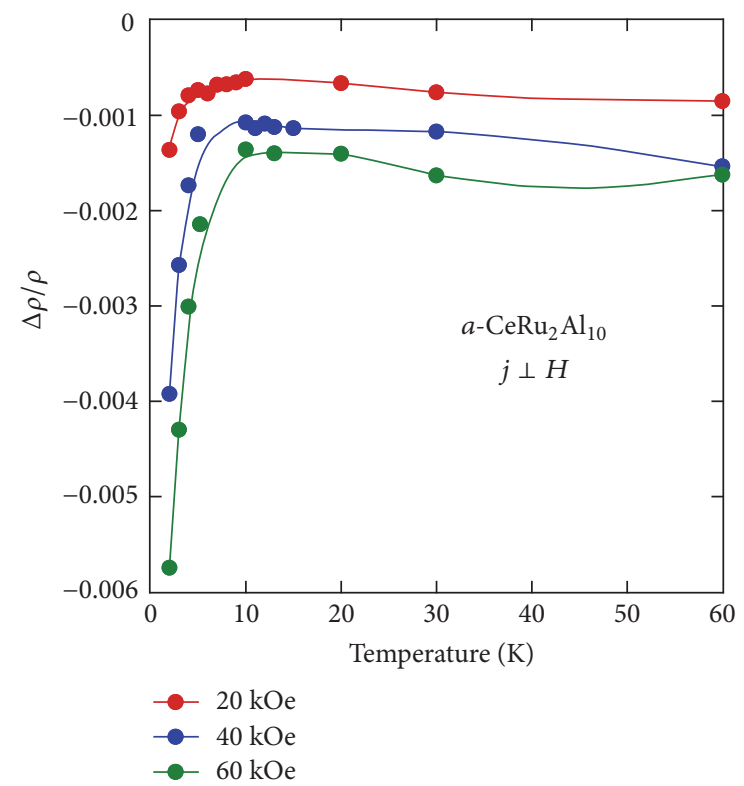

FIGURE 6: Temperature dependence of the magnetoresistance $\Delta \rho / \rho$ under various magnetic fields for $a-\mathrm{CeRu}_{2} \mathrm{Al}_{10}$ alloy.

the amorphous alloy is generally considered to be similar to that of the crystalline counterpart. Even in $a-\mathrm{CeRu}_{2} \mathrm{Al}_{10}$ alloy, a local structure similar to that of the crystalline $\mathrm{CeRu}_{2} \mathrm{Al}_{10}$ is expected to be realized. In addition, based on $\mu_{\text {eff }}$, we have shown that half of $\mathrm{Ce}$ in $a$-CeRu$u_{2} \mathrm{Al}_{10}$ alloy is in the trivalent state. $\mathrm{Ce}^{3+}$ ions in $a-\mathrm{CeRu}_{2} \mathrm{Al}_{10}$ alloy are distributed randomly in the amorphous sample. In this case, it is considered that the random distribution of $\mathrm{Ce}^{3+}$ in the disordered structure realizes the impurity Kondo effect at low temperatures. Thus, the enhancement of $\rho$ and $C_{p} / T$ is observed in the low-temperature region for $a$-CeRu${ }_{2} \mathrm{Al}_{10}$ alloy. Since the Kondo scattering is suppressed by the applying a magnetic field, $\Delta \rho / \rho$ increases negatively. Therefore, the negative increase of $\Delta \rho / \rho$ for $a$-CeRu $\mathrm{Cl}_{10}$ alloy at low temperatures and high magnetic fields indicates that the scattering center of the resistivity is due to Kondo scattering.

\section{Conclusion}

We prepared $a$-CeRu${ }_{2} \mathrm{Al}_{10}$ and $a-\mathrm{LaRu}_{2} \mathrm{Al}_{10}$ alloys and measured their susceptibility, resistivity, and specific heat. $\chi$ value for $a-\mathrm{CeRu}_{2} \mathrm{Al}_{10}$ alloy follows the Curie-Weiss law in the high-temperature region of $T>30 \mathrm{~K}$ with no magnetic transition. The effective paramagnetic moment and the Weiss temperature are $1.19 \mu_{B} / \mathrm{Ce}$-atom and $-20 \mathrm{~K}$, respectively. $\rho$ value for $a-\mathrm{CeRu}_{2} \mathrm{Al}_{10}$ and $a-\mathrm{LaRu}_{2} \mathrm{Al}_{10}$ alloys shows a small temperature dependence. However, an enhancement of $\rho$ was observed in the low-temperature region for $a$-CeRu $\mathrm{Al}_{10}$ alloy. $C_{p} / T$ value of $a-\mathrm{CeRu}_{2} \mathrm{Al}_{10}$ alloy increases rapidly with decreasing $T^{2}$ below $100 \mathrm{~K}^{2}$. The magnetoresistance $\Delta \rho / \rho$ for $a-\mathrm{CeRu}_{2} \mathrm{Al}_{10}$ alloy increases rapidly with decreasing temperature for $T<10 \mathrm{~K}$. Therefore, it is considered that $a$ $\mathrm{CeRu}_{2} \mathrm{Al}_{10}$ alloy is formed the impurity Kondo state in the low-temperature region.

\section{Conflicts of Interest}

The authors declare that there are no conflicts of interest regarding the publication of this paper.

\section{Acknowledgments}

The authors would like to thank Dr. S. Yamanaka of the Muroran Institute of Technology for allowing the use of the SEM-EDS. The authors wish to thank Dr. Y. Kawamura of the Muroran Institute of Technology for the useful discussion on crystalline $\mathrm{CeRu}_{2} \mathrm{Al}_{10}$.

\section{References}

[1] A. M. Strydom, "Thermal and electronic transport in $\mathrm{CeRu}_{2} \mathrm{Al}_{10}$ : evidence for a metal-insulator transition," Physica B: Condensed Matter, vol. 404, no. 19, pp. 2981-2984, 2009.

[2] T. Nishioka, Y. Kawamura, T. Takesaka et al., "Novel phase transition and the pressure effect in $\mathrm{YbFe}_{2} \mathrm{Al}_{10}$-type $\mathrm{CeT}_{2} \mathrm{Al}_{10}$ ( $\mathrm{T}=\mathrm{Fe}, \mathrm{Ru}, \mathrm{Os})$," Journal of the Physical Society of Japan, vol. 78, no. 12, Article ID 123705, 2009.

[3] H. Tanida, D. Tanaka, M. Sera et al., "Possible long-range order with singlet ground state in $\mathrm{CeRu}_{2} \mathrm{Al}_{10}$," Journal of the Physical Society of Japan, vol. 79, no. 4, Article ID 043708, 2010.

[4] H. Tanida, D. Tanaka, M. Sera, T. Nishioka, and M. Matsumura, "Long range order in CeRu2Al10," Journal of the Physical Society of Japan, vol. 80, Article ID SA023, 2011.

[5] Q. S. Zeng, C. R. Rotundu, W. L. Mao et al., "Low temperature transport properties of Ce-Al metallic glasses," Journal of Applied Physics, vol. 109, no. 11, Article ID 113716, 2011.

[6] Y. Amakai, S. Murayama, Y. Obi, H. Takano, and K. Takanashi, "Evidence of a heavy fermion state in the disordered Ce-alloy 
system without translation symmetry," Physical Review B Condensed Matter and Materials Physics, vol. 79, no. 24, Article ID 245126, 2009.

[7] Y. Amakai, S. Murayama, Y. Obi, H. Takano, N. Momono, and K. Takanashi, "Thermal expansion of structure-disordered heavyfermion Ce alloys," Journal of the Physical Society of Japan, vol. 80, Article ID SA057, 2011.

[8] Y. Obi, S. Murayama, Y. Amakai, Y. Okada, and K. Asano, "Heavy fermion like behavior and superconductivity in amorphous $\mathrm{Ce}_{x} \mathrm{Ru}_{100-x}$ alloy," Physica B: Condensed Matter, vol. 378380, pp. 857-858, 2006.

[9] Y. Amakai, S. Murayama, Y. Obi, H. Takano, N. Momono, and K. Takanashi, "Magnetic properties of structure-disordered heavy fermion Ce-Ru alloys," Journal of Physics: Conference Series, vol. 150, no. 4, Article ID 042004, 2009.

[10] Y. Amakai, D. Yoshii, S. Murayama et al., "La substitution effect to the heavy-fermion state in structure-disordered Ce$\mathrm{Ru}$ alloys," Journal of Physics: Conference Series, vol. 391, no. 1, Article ID 012002, 2012.

[11] W. Yang, H. Liu, X. Yang, and L. Dou, "Low temperature specific heat of amorphous alloys," Journal of Low Temperature Physics, vol. 160, 148 pages, 2010. 

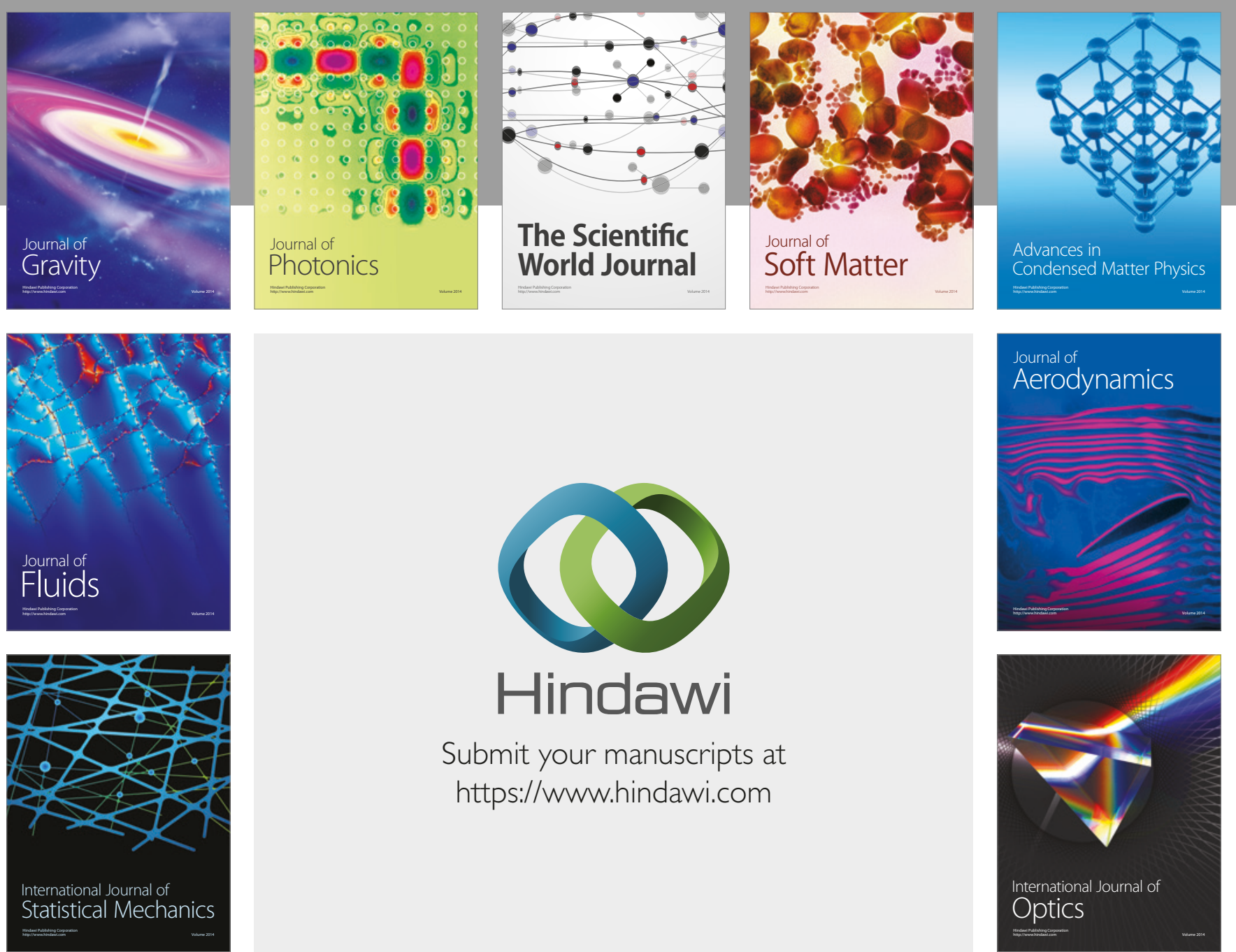

Submit your manuscripts at

https://www.hindawi.com
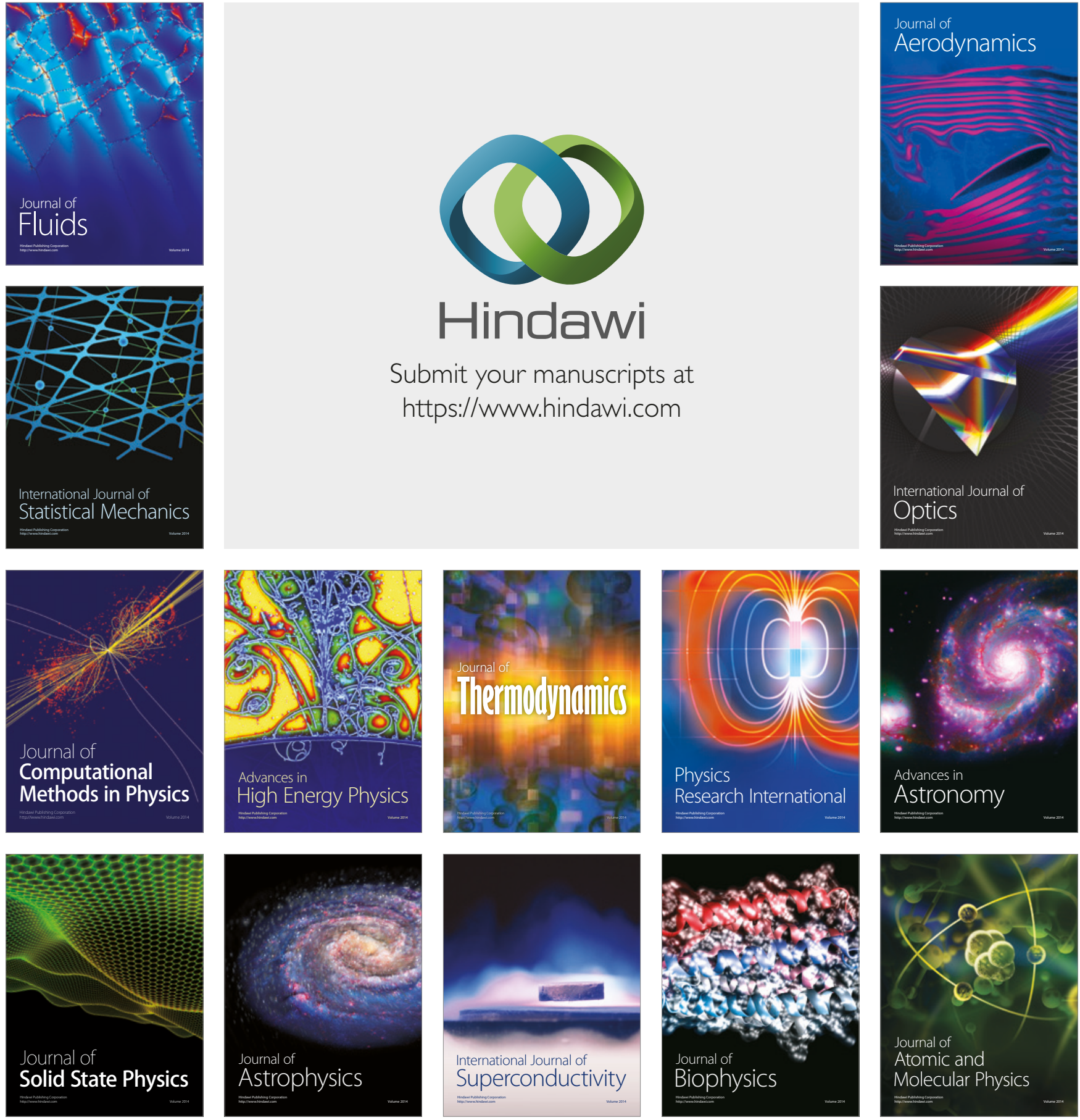